\title{
Denoising the Denoisers: An independent evaluation of microbiome sequence error-correction approaches
}

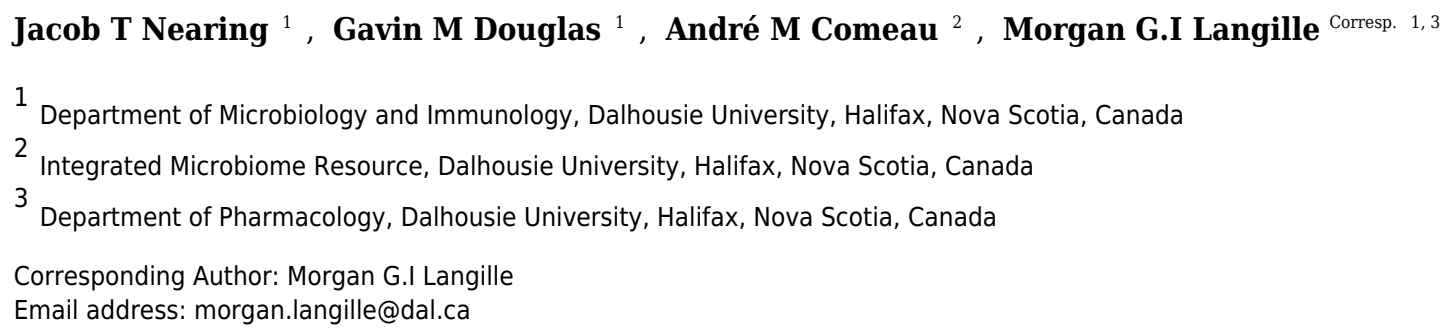

High-depth sequencing of universal marker genes such as the 16S rRNA gene are a common strategy to profile microbial communities. Traditionally, sequence reads are clustered into operational taxonomic units (OTUs) at a defined identity threshold to avoid sequencing errors generating spurious taxonomic units. However, there have been numerous bioinformatic packages recently released that attempt to correct sequencing errors to determine real biological sequences at single nucleotide resolution by generating amplicon sequence variants (ASVs). As more researchers begin to use high resolution ASVs, there is a need for an in-depth and unbiased comparison of these novel "denoising" pipelines. In this study, we conduct a thorough comparison of three of the most widelyused denoising packages (DADA2, UNOISE3, and Deblur) as well as an open-reference $97 \%$ OTU clustering pipeline on mock, soil, and host-associated communities. We found from the mock community analyses that although they produced similar microbial compositions based on relative abundance, the approaches identified vastly different numbers of ASVs that significantly impact alpha diversity metrics. Our analysis on real datasets using recommended settings for each denoising pipeline also showed that the three packages were consistent in their per-sample compositions, resulting in only minor differences based on weighted UniFrac and Bray-Curtis dissimilarity. DADA2 tended to find more ASVs than the other two denoising pipelines when analyzing both the real soil data and two other host-associated datasets, suggesting that it could be better at finding rare organisms, but at the expense of possible false positives. The open-reference OTU clustering approach identified considerably more OTUs in comparison to the number of ASVs from the denoising pipelines in all datasets tested. The three denoising approaches were significantly different in their run times, with UNOISE3 running greater than 1200 and 15 times faster than DADA2 and Deblur, respectively. Our findings indicate that, although all pipelines result in similar general community structure, the number of ASVs/OTUs and 
resulting alpha-diversity metrics varies considerably and should be considered when attempting to identify rare organisms from possible background noise. 


\section{Denoising the Denoisers: An independent evaluation of microbiome}

\section{3 sequence error-correction approaches}

4

5

6 Jacob T. Nearing ${ }^{1}$, Gavin M. Douglas ${ }^{1}$, André M. Comeau², Morgan G. I. Langille ${ }^{1,2,3}$

7

$8{ }^{1}$ Department of Microbiology \& Immunology, Dalhousie University, Halifax, NS, Canada

9

$10{ }^{2}$ Integrated Microbiome Resource, Dalhousie University, Halifax, NS, Canada

11

$12{ }^{3}$ Department of Pharmacology, Dalhousie University, Halifax, NS, Canada

13

14

15 Corresponding Author:

16

17 Morgan Langille

18

19

20

21 Email address: morgan.langille@dal.ca

22 


\section{Abstract}

High-depth sequencing of universal marker genes such as the 16S rRNA gene are a common strategy to profile microbial communities. Traditionally, sequence reads are clustered into operational taxonomic units (OTUs) at a defined identity threshold to avoid sequencing errors generating spurious taxonomic units. However, there have been numerous bioinformatic packages recently released that attempt to correct sequencing errors to determine real biological sequences at single nucleotide resolution by generating amplicon sequence variants (ASVs). As more researchers begin to use high resolution ASVs, there is a need for an in-depth and unbiased comparison of these novel "denoising" pipelines. In this study, we conduct a thorough comparison of three of the most widely-used denoising packages (DADA2, UNOISE3, and Deblur) as well as an open-reference 97\% OTU clustering pipeline on mock, soil, and hostassociated communities. We found from the mock community analyses that although they produced similar microbial compositions based on relative abundance, the approaches identified vastly different numbers of ASVs that significantly impact alpha diversity metrics. Our analysis on real datasets using recommended settings for each denoising pipeline also showed that the three packages were consistent in their per-sample compositions, resulting in only minor differences based on weighted UniFrac and Bray-Curtis dissimilarity. DADA2 tended to find more ASVs than the other two denoising pipelines when analyzing both the real soil data and

42 two other host-associated datasets, suggesting that it could be better at finding rare organisms, 43 but at the expense of possible false positives. The open-reference OTU clustering approach 44 identified considerably more OTUs in comparison to the number of ASVs from the denoising 45 pipelines in all datasets tested. The three denoising approaches were significantly different in 
46 their run times, with UNOISE3 running greater than 1200 and 15 times faster than DADA2 and

47 Deblur, respectively. Our findings indicate that, although all pipelines result in similar general

48 community structure, the number of ASVs/OTUs and resulting alpha-diversity metrics varies

49 considerably and should be considered when attempting to identify rare organisms from possible

50 background noise.

51

\section{Introduction}

53

54 Microbiome studies often use an amplicon sequencing approach where a single genomic region 55 is sequenced at a sufficient depth to provide relative abundance profiles of the microbes present 56 in a sample. The $16 \mathrm{~S}$ rRNA gene (16S) is usually chosen as a marker gene for sequencing of

57 bacterial communities due to its unique structure that contains both conserved and variable 58 regions and presence in all known Bacteria and Archaea species. This sequencing approach is 59 often used to avoid the high cost of shotgun metagenomic sequencing or to avoid problems with 60 sequencing non-microbial DNA from host contamination. However, sequencing errors make it 61 difficult to distinguish biologically real nucleotide differences in $16 \mathrm{~S}$ sequences from sequencing

62 artefacts. To avoid this issue sequences are often clustered into operational taxonomic units 63 (OTUs) at a particular identity threshold (e.g. 97\%) to avoid the problem of differentiating 64 biological from technical sequence variations; however, this comes at the cost of taxonomic 65 resolution. Recently, many new bioinformatic sequence "denoising" approaches have been 66 developed to address this issue by attempting to correct sequencing errors thus improving 67 taxonomic resolution. These pipelines differ in how they correct sequencing errors. DADA2 68 generates a parametric error model that is trained on the entire sequencing run and then applies 
69 that model to correct and collapse the sequence errors into what the authors call amplicon

70 sequence variants (ASVs) (Callahan et al., 2016). This approach is advantageous as it builds

71 unique error models for each sequencing run. Deblur aligns sequences together into "sub-OTUs"

72 and, based on an upper error rate bound along with a constant probability of indels and the mean

73 read error rate, removes predicted error-derived reads from neighboring sequences (Amir et al.,

74 2017). Deblur employs a sample-by-sample approach which reduces both memory requirements

75 and computational demand. UNOISE3 uses a one-pass clustering strategy that does not depend

76 on quality scores, but rather two parameters with pre-set values that were curated by its author to

77 generate "zero-radius OTUs" (Edgar, 2016). The advantage of a one-pass clustering strategy is

78 that it saves on the computational time required to analyze the sequences in the provided study.

79 Note that ASVs, sub-OTUs, and zero-radius OTUs are synonymous and the term ASV will be

80 used henceforth. Denoising approaches provide improved resolution and they avoid having to

81 make a choice between various OTU strategies which may result in differing results (Edgar,

82 2017). In addition, ASVs can be identified by their unique biological sequences instead of

83 relying on per-study IDs, which allows for easier comparison across datasets (Callahan,

84 McMurdie \& Holmes, 2017).

85 Although there have been several bioinformatic comparisons of OTU-based approaches

86 (Allali et al., 2017; Plummer \& Twin, 2015), a thorough third-party comparison of denoising

87 pipelines has yet to be conducted. In this paper, we compare the strengths and weaknesses of the

88 DADA2, UNOISE3, and Deblur packages along with a comparison to an open-reference $97 \%$

89 OTU-based approach (Rognes et al., 2016), while following the recommended default settings.

90 We assess the accuracy of these approaches using several mock communities including both 
91 bacterial and fungal amplicons. In addition, we compare the results of the four pipelines on three

92 previously-published real human, mouse, and soil datasets.

$94 \underline{\text { Methods }}$

95 Sequence Acquisition

96 The HMP mock community (which has even expected abundances) and the ZymoBIOMICS

97 Microbial Community Standard (referred to as the Zymomock community) were sequenced by

98 the Integrated Microbiome Resource at Dalhousie University using an Illumina MiSeq on

99 separate sequencing runs, as previously described using the V4-V5 16S rRNA gene region

100 (Comeau et al., 2017). Reads were then uploaded to the European Nucleotide Archive (ENA)

101 under accession number PRJEB24409. The Extreme dataset (mock-12) originally presented in

102 the DADA2 paper and the fungal ITS1 dataset (mock-9) were retrieved from the Mockrobiota

103 project (Bokulich et al., 2016). The Extreme dataset was sequenced using an Illumina MiSeq

104 (Callahan et al., 2016) and the fungal mock community was sequenced using an Illumina HiSeq

105 (Bokulich et al., 2016). The soil data set collected from blueberry fields (available under NCBI

106 SRA PRJNA389786) and the exercise dataset collected from stool of mice that exercised plus

107 controls (ENA accession PRJEB18615) (Lamoureux et al., 2017) as well as the human-

108 associated dataset of intestinal biopsies of pediatric Crohn's disease patients plus controls (ENA

109 accession PRJEB21933) (Douglas et al., 2018) were sequenced at the Integrated Microbiome

110 Resource at Dalhousie University.

112 Filtering 
113 All sample data was filtered using the Microbiome Helper filtering scripts (Comeau et al., 2017).

114 In summary, primers were trimmed off all reads using Cutadapt (v 1.14) (Martin, 2011) and

115 GNU Parallel (Tange, 2011). Primer-free sequences were then input into the dada2_filter.R

116 script available in Microbiome Helper. This script takes in the maximum expected number of

117 errors allowed as well as a truncation length. The HMP mock community and the Zymomock

118 community were truncated to read lengths of 270 and 210 base pairs for the forward and reverse

119 reads respectively to remove low quality bases at the end of the reads. The shorter length for the

120 reverse reads is a result of their lower overall quality compared to the forward reads. Note that

121 for DADA2, and Deblur to work properly reads need to be of the same length. The single-end

122 reads from the Extreme mock community and the fungal mock community were truncated to 80

123 base-pairs. The three real datasets: soil, mouse, and human-associated, were truncated to 270 and

124210 base-pairs for the forward and reverse reads, respectively. The number of expected errors

125 allowed were defined as three different filtering stringencies: 5 (low), 3 (medium), and 1 (high).

126

127 DADA2 Pipeline

128 The DADA2 pipeline was run using Microbiome Helper scripts, which wraps the core

129 algorithms of the DADA2 pipeline (Callahan et al., 2016). Filtered reads were input into the 130 wrapper script dada2_inference.R which runs the DADA2 inference algorithm. Once ASVs are 131 determined, they are passed into DADA2's chimera-checking algorithm which was run using the 132 wrapper script dada2_chimera_taxa.R to screen out chimeric sequences. The output objects 133 containing ASV sequences and abundances counts were then converted into BIOM table format 134 using convert_dada2_out.R. All DADA2 wrapper scripts were run with default settings. 135

136 UNOISE3 Pipeline 
137 Filtered reads were input into USEARCH's (v 10) (Edgar, 2010) fastq_mergepairs command if

138 they were paired-end reads or concatenated together into one FASTQ if they were single-end

139 reads. Next, the merged FASTQ file was converted into a FASTA file using the Microbiome

140 Helper script run_fastq_to_fasta.pl and then used as input for USEARCH's fastx_uniques

141 command which generated a FASTA containing all the unique sequences and their abundance.

142 Finally, the FASTA containing unique sequences was used as input into USEARCH's unoise3

143 (Edgar, 2016) command generating a BIOM table and representative ASVs that were used in

144 subsequent analyses. All USEARCH scripts were run with default settings.

145

146 Deblur Pipeline

147 Paired-end filtered reads were stitched together using the Microbiome Helper wrapper script

148 run_pear.pl which wraps the program PEAR (v 0.9.10) (Zhang et al., 2014). This step was

149 skipped for filtered single-end reads. Next, reads were renamed to match a format that was

150 compatible with QIIME2 (Caporaso et al., 2010b) and converted into a QIIME2 artifact. Samples

151 were then run through QIIME2's built-in deblur command using the $16 \mathrm{~S}$ setting, which uses

152 Greengenes 13_8 (DeSantis et al., 2006) for positive filtering. A separate analysis to test the

153 effect of positive filtering was conducted by using the non-positive filtered output files

154 ("all.biom" and "all.seqs.fa") from the stand alone version of Deblur. Fungal reads were run

155 using the "other" setting and the UNITE 10.10.2017 database (Kõljalg et al., 2013). Finally, the

156 representative ASV sequences and a BIOM table were exported from the QIIME2 artifact. All

157 other Deblur scripts were run with default settings.

158

159 Open Reference OTU Pipeline 
160 Filtered reads were stitched and imported into a QIIME2 artifact as previously described in the

161 Deblur pipeline. The imported sequences were then dereplicated using the QIIME2 VSEARCH

162 plugin. Dereplicated sequences were filtered for chimeras using the QIIME2 VSEARCH

163 UCHIME reference-based chimera filtering (Edgar et al., 2011) using the Greengenes13_8 97\%

164 OTU database for reference. Chimera-checked sequences were then clustered at $97 \%$ in an open-

165 reference fashion using the QIIME2 VSEARCH cluster-features-open-reference plugin. The

166 Greengenes 13_8 97\% OTU database was used as the reference database during open-reference

167 OTU picking. Finally, singleton OTUs were removed from the OTU table and the table was

168 exported in BIOM format along with its representative sequences in FASTA format.

169

170 Run Time and Memory Analysis

171 The soil dataset was filtered using the low-stringency filter and then individual samples were

172 rarefied to either 5000, 10000, 20000 or 30000 reads per sample. The different read-depth sets

173 were then run through the three denoising pipelines and user time and maximum memory usage

174 was determined using the GNU time (v 1.7) command.

175

176 ASV and OTU Analysis of Mock Communities

177 ASVs and OTUs were compared against the expected sequences provided with each of the mock

178 communities. This comparison was done using the command-line BLASTN (v 2.7.10) (Altschul

179 et al., 1990) tool against the expected sequences from each community. This allowed us to

180 determine the number of full length 100\% matches and 97\% matches. All ASVs and OTUs that

181 did not match these criteria were then compared against the SILVA 16S rRNA gene database (v

182 128) (Pruesse et al., 2007) or the UNITE 10.10.2017 database to find all full length 100\% and 
$18397 \%$ matches. Any ASVs or OTUs that did not match these databases were then labeled as

184 "Unmatched". The number of unique expected sequences for each mock community was

185 determined by slicing out the amplified regions using a custom Python (v 3.6.1) script

186 (slice_amplified_region.py, available on GitHub) from the expected sequences of each mock

187 community and then finding the number of unique sequences from this output using

188 USEARCH10's fastx_uniques command. In addition to analysis done with no abundance

189 filtering, we also compared how filtering of low abundance ASVs/OTUs affected the type and

190 amount of ASVs/OTUs called by each pipeline. This was done by applying a $0.1 \%$ minimum

191 abundance filter to each approach and dataset except for the Extreme mock community where a

$1920.0004 \%$ minimum abundance filter was applied. The $0.1 \%$ minimum abundance filter was

193 chosen based on the known $0.1 \%$ bleed through between Illumina MiSeq runs. A lower

194 minimum abundance filter of $0.0004 \%$ was chosen for the Extreme mock community since some

195 of the expected sequences had a lower expected abundance than $0.1 \%$. Histograms with

196 sequence identity to the expected sequences were generated for each pipeline and each mock

197 community except for the fungal mock community due to the community missing some expected

198 sequences from its expected sequence list. All sequences that showed less than $75 \%$ identity

199 were binned together.

200

201 Abundance Data Analysis of Mock Communities

202 For the HMP, Zymomock and Extreme datasets all ASVs/OTUs that matched at 97\% identity or

203 greater with the provided expected sequences based on a BLASTN search were added to the

204 abundance of the corresponding expected taxa. Stacked bar charts of expected taxa relative

205 abundances were created using the ggplot2 (v 2.2.1) (Wickham, 2009) R (v 3.4.3) (R 
206 Development Core Team, 2008) package and the cowplot (v 0.9.2) R package (see Data

207 Deposition for exact scripts).

208 Due to the incomplete nature of the expected sequences for the fungal mock community,

209 UNITE database hits at $97 \%$ or greater to an expected species within the community were

210 considered as expected ASVs/OTUs. All other ASVs/OTUs were classified as "Non-Reference"

211 hits.

212

213 Analysis of Real Datasets

214 Sequences from the three real datasets; soil, human-associated, and exercise were filtered using

215 medium stringencies (allowing up to 3 expected errors per sequence) for each package and

216 rarified to 5215, 3000 and 4259 reads, respectively. These rarefaction levels were chosen as they

217 were the lowest read count above 2000, after the DADA2 pipeline was complete. ASV

218 abundance tables output by all approaches were combined into a single table where each

219 biological sample was represented multiple times (once for each pipeline). ASVs/OTUs not

220 called by a specific pipeline were given an abundance of zero in their column (e.g. ASVs only

221 called by Deblur for sampleA were given zero abundances in the columns for DADA2's and

222 UNOISE3's outputs of sampleA). Representative sequences were aligned against the Greengenes

223 13_5 99\% OTU aligned sequences and placed on the Greengenes 13_599\% OTU tree using

224 SEPP (Mirarab et al., 2011). Weighted UniFrac and unweighted UniFrac distances at the

225 ASV/OTU level were then generated using the QIIME1 beta_diversity.py command and

226 principal coordinates were generated using the QIIME1 prinicpal_cordinates.py command.

227 The Bray-Curtis distance matrix at the genus level was generated by assigning taxonomy

228 to the resulting ASV/OTUs from each package using the RDP classifier (Cole et al., 2014) with

229 the assignTaxonomy function available in the DADA2 package and the rdp_train_set_16 
230 database. Distances were then generated using the summarize_taxa.py and beta_diversity.py

231 commands in QIIME1. Ordination was generated using the metaMDS function in the vegan $\mathrm{R}$

232 package (Oksanen et al., 2018).

233 Mantel correlations between each distance matrix for each pipeline and each beta-

234 diversity metric (weighted/unweighted UniFrac and Bray-Curtis) were generated using the vegan

235 R package. The number of observed OTUs/ASVs per sample for each real dataset were

236 generated using the rarified combined OTU/ASV tables and the alpha_diversity.py command in

237 QIIME1 using observed OTUs as the metric option.

238

$239 \underline{\text { Results }}$

240

241 Differences in methodology and availability between different denoising pipelines

242 There are important aspects other than accuracy that need to be considered when determining

243 which denoising package a researcher should use for their project. Both DADA2 and UNOISE3

244 are suggested to be run in a pooled-sample workflow, where all sequences are pooled together

245 during the denoising process (Table 1). This allows them to better account for batch errors

246 across multi-run experiments. Deblur, on the other hand, runs its denoising process sample-by-

247 sample. This approach helps lower Deblur's computational requirements, but at the cost of

248 reducing its ability to correct multi-run batch effects. Both DADA2 and Deblur are open source

249 projects, whereas UNOISE3 is a closed-source project which has a free 32-bit academic version

250 with a $4 \mathrm{~Gb}$ memory cap and a full 64-bit version that costs between \$885-1485 USD (Table 1).

251 Another major difference is that the built-in Deblur function in QIIME2 has a positive filtering

252 process. This default setting causes Deblur to discard reads that do not reach a length-scaled bit 
253 score and an e-value threshold to any sequence in the $88 \%$ representative sequences Greengenes

254 database. Note the default database can be changed using the "other" version of the Deblur

255 plugin in QIIME2, an important feature when working with fungal or eukaryotic data. It is also

256 important to note that the stand-alone version of Deblur outputs both positively filtered and non-

257 filtered results by default, unlike the QIIME2 plugin which, only outputs the positively filtered

258 results. Currently, the functionality of both DADA2 and Deblur can be accessed through a

259 graphical user interface as plugins in QIIME2 Studio, whereas UNOISE3 does not support a

260 graphical user interface (Table 1).

261

262 Total number of ASVs/OTUs vary across approaches in mock communities

263 We processed four different mock communities with the DADA2, UNOISE3, and Deblur

264 denoising pipelines as well as an open-reference 97\% OTU clustering pipeline to compare the

265 resulting ASVs/OTUs from each approach. Focusing on the number of called ASVs from each

266 denoising pipeline, we found no approach consistently called more ASVs. DADA2 called the

267 most ASVs in two mock communities (HMP: 42, Extreme: 78) and UNOISE3 called the most

268 ASVs in the other two mock communities (Fungal: 38, Zymomock: 43) under medium

269 stringency filtering (Fig 1, Supp Table 1). Overall, open-reference OTU clustering output the

270 most ASVs/OTUs for all four mock communities (HMP: 453, Extreme: 8891, Fungal: 96,

271 Zymomock: 294). None of the approaches were capable at outputting all expected sequences at

$272100 \%$ identity in any of the mock communities that were processed and, in all datasets, at least

273 one denoising pipeline output more ASVs than expected sequences. All four approaches output

274 at least one ASV/OTU at $97 \%$ or greater identity from all organisms in the HMP mock

275 community and the Zymomock community (Supp Table 2,3). DADA2 output nine more ASVs 
276 with $97 \%$ or greater identity matches to expected sequences in the Extreme dataset (read depth of

27711.6 million reads) than the other two denoising pipelines (Supp Table 4). Six of the nine taxa

278 that DADA2 called and the other pipelines did not call, had expected relative abundances of only

$2790.000427 \%$ (Supp Table 4). Of the other three taxa not found by Deblur or UNOISE3, one was

280 at an expected abundance of $0.00427 \%$ and two had an expected abundance of $0.0427 \%$ (Supp

281 Table 4). Interestingly, open reference OTU clustering called OTUs corresponding to these nine

282 expected taxa along with two more expected taxa that DADA2 missed, both of which were in the

283 lowest possible expected abundance range (0.000427\%). One organism was missed by all

284 pipelines in the Extreme community (C. methylpentusum), which was also in the lowest possible

285 expected abundance range. Sequence identity histograms were constructed for the HMP,

286 Zymomock and Extreme communities (Supp Fig 1-3). In the HMP community, no ASV was

287 found to have below $80 \%$ identity by the denoising pipelines, but open reference OTU clustering

288 found 21 OTUs below 80\% identity. Furthermore, the OTU pipeline called 324 OTUs below

289 99\% identity whereas all denoising approaches called less than 15 ASVs (Supp Fig 1). In the

290 Extreme community, most ASVs were found in the 99-100\% identity range whereas the majority

291 of OTUs found by open-reference OTU clustering were in the 95-97\% identity range (Supp Fig

292 2).

293

Due to the expected sequences for the fungal community being incomplete, we included

294

any UNITE database hits as expected sequences if the matching sequence was from a species

295 that was included in the fungal community. This resulted in almost all the fungi present in the

296 community to be found by all four pipelines except for Penicillium allii and $P$. commune. Open-

297 reference OTU clustering did find P. allii, although at an Extremely low abundance of 0.004\%

298 compared to its expected abundance of $6.25 \%$. 
Given that some of the above potential spurious ASVs would be removed by sequence

300 bleed-through (Illumina, 2017) or low-abundance filters in typical workflows, we applied an

301 abundance cutoff filter of $0.1 \%$ to each mock community except for the Extreme community,

302 where an abundance filter of $0.0004 \%$ was applied (Supp Fig 4). This lower abundance filter

303 was chosen due to some expected sequences being in abundance of $0.000427 \%$. Application of

304 the abundance filter to the HMP community resulted in all 10 unmatched ASVs (those that did

305 not match either the expected or SILVA by $97 \%$ or greater) called by DADA2 to be discarded,

306 but none of the four unmatched reads in UNOISE3 to be discarded (Supp Fig 4). A similar

307 phenomenon was seen in the Zymomock community with all 12 of Deblur's unmatched reads

308 being discarded (along with one database hit) and UNOISE3 only discarding one of 19

309 unmatched reads it called. The largest effect that the application of the filters had was the

310 removal of a significant amount of OTUs found in each mock community by the open-reference

311 OTU pipeline. The most drastic change was seen in the Extreme community that started with

3128891 OTUs and was reduced to 1248 OTUs (Supp Fig 4). In the fungal community the number

313 of OTUs found by open-reference OTU clustering (27) became less than the number of ASVs

314 found by UNOISE3 (36).

315 To determine how read quality filtering affects the number of ASVs called by each

316 pipeline, we ran each denoising pipelines using two additional quality filtering stringencies, low

317 and high (see Methods). The different quality filter stringencies used made only small impacts on

318 the numbers of ASVs called by each pipeline for the HMP, Extreme and fungal datasets (Supp

319 Table 1). A difference of six ASVs was the largest between the high and medium stringencies on

320 the HMP community and was output by UNOISE3 (Supp Table 1). In the Zymomock

321 community, the number of ASVs called by DADA2 only varied by one for all three stringencies, 
322 but Deblur varied by as much as 12 ASVs and UNOISE3 varied by as much as 16 ASVs being

323 outputted between the high and medium filter stringencies (Supp Table 1).

324

325 Denoising pipelines are consistent in determining mock community composition

326 Despite the different ASV counts between each pipeline in the mock community, the relative

327 abundances of the expected taxa are strikingly similar (Fig. 2). In both the HMP and Zymomock

328 communities, only a small portion of ASVs called by DADA2 and Deblur did not match the

329 expected sequences by $97 \%$ identity or greater. In contrast, UNOISE3 identified multiple (8 in

330 HMP, 20 in Zymomock) sequences that summed together to make up $2.9 \%$ and $4.8 \%$ of the

331 relative abundance in the HMP and Zymomock communities, respectively (Supp Table 1,2,3).

332 Open-reference OTU clustering found 6783 non-reference OTUs (Supp Table 1) in the Extreme

333 community that summed together to make up $2.6 \%$ of the community, whereas the denoising

334 approaches all found non-reference abundances less than or equal to $0.3 \%$ (Supp Table 4). None

335 of the approaches were good at distinguishing the proper abundances of the two Parabacteroides

336 distasonis strains with denoising pipelines finding similar proportions of both strains and the

337 open-reference OTU pipeline finding dominance of the 13400 strain and not the 13401 strain

338 (Fig 2b, Supp Table 4). None of the approaches performed well at matching the expected

339 abundance of the Zymomock community or the fungal community (Fig 2). All three denoising

340 pipelines called over-abundances of Lactobacillus fermentum in the Zymomock community,

341 with Deblur calling the most (44.5\%) and UNOISE3 calling the least (37.6\%) (Supp Table 3).

342 Similarly, all approaches called non-reference hits in greater than $9 \%$ abundance in the fungal

343 community (Supp Table 5), with UNOISE3 calling the most non-reference hits (12.4\%) and

344 Deblur calling the least $(9.8 \%)$. Due to all four pipelines producing similar proportions of non- 
345 reference hits in the fungal community, this could suggest that either the mock compositions are

346 not in the expected proportions, the four pipelines are similarly biased, or that an upstream

347 process during sequencing caused the introduction of unexpected sequences.

348

349 Weighted beta-diversity results from different approaches are indistinguishable in real soil 350 and host-associated communities

351 After comparing each pipeline using mock communities, we next wanted to investigate how

352 comparable the results between pipelines were for real 16S datasets. We compared the pipelines

353 on a soil dataset (soil) due to its high diversity (Fierer \& Jackson, 2006) a mouse exercise stool

354 dataset (exercise) and a gut biopsy sample dataset from Crohn's Disease patients plus controls

355 (human-associated). The intra-sample distances based on weighted UniFrac measurements were

356 comparable between each approach among all three datasets (Fig 3). In general, we found the

357 intra-samples distances to be small, ranging between median values of $\sim 0.09$ and $\sim 0.15$. There

358 were no consistent differences seen between all three datasets. Looking at the soil dataset, we

359 found that all three denoising pipelines had similarly small intra-sample distances $(\sim 0.11)$ (Fig

360 3A), while the open-reference OTU clustering pipeline was slightly further away from UNOISE3

361 and Deblur ( 0.13). Despite this observation, it is important to note that this difference is still

362 relatively small. Furthermore, when each sample was plotted onto a PCoA we found that samples

363 tended to group by biological origin rather than the approach used to process the sequences,

364 suggesting that a relatively similar PCoA plot would be generated by each pipeline (Fig 3D-F).

365 The Mantel correlations between each weighted UniFrac distance matrix were all highly

366 correlated (correlation values ranging from 0.764 to 0.975 ), which suggests similar weighted

367 UniFrac profiles between each approach (Supp Table 6-8). 
we found that intra-sample distances based on genus-level assignment were similar among different approaches (Supp Fig 5). In general, the intra-sample distances were relatively low, with median values ranging from $\sim 0.04$ to $\sim 0.20$, indicating a high amount of agreement between approaches. Interestingly, we found that the intra-sample distances seemed to increase across datasets based on how diverse the samples within them were (Supp Fig 5). In two of the three datasets (soil and BISCUIT), we found that DADA2 and UNOISE3 tended to be closer together in distance than Deblur was to DADA2 or UNOISE3, indicating a slightly higher amount of agreement between DADA2 and UNOISE3 at the genus level. Again, these differences were relatively small and would have minimal impacts on biological results obtained from them.

378 Plotting the Bray-Curtis dissimilarity matrices onto an NMDS plot resulted in similar findings as 379 weighted UniFrac, with samples grouping by biological origin rather than the pipeline used to

process them. Furthermore, we found that the Bray-Curtis dissimilarity matrices were extremely well correlated with each other, ranging in values between 0.956-0.995 (Supp Table 6-8).

We next compared unweighted UniFrac distances, which is a metric that considers the presence or absence of ASVs/OTUs and their phylogenetic distance. We found that the median intra-sample distance between each pipeline was much greater, ranging between $\sim 0.40$ to $\sim 0.79$ (Supp Fig 6). Similar to our results focused on Bray-Curtis dissimilarity, we also found that the median intra-sample distance between samples tended to increase with sample diversity (0.720.79 for soil; $0.40-0.55$ for human-associated; $0.60-0.70$ for exercise. The PCoA plot based on these distances resulted in samples grouping together based on pipeline, rather than biological origin, indicating large differences between the different approaches (Supp Fig 6). 
To look at how much of a difference the positive filtering process had on the profiles

391

392

393

394

395

396

397

398

399

400

401

402

403

404

405

406

407

408

409

410

411

412

generated by Deblur and to see if the slight difference in Bray-Curtis dissimilarity among the denoising approaches was caused by this filter, we ran the stand-alone version of Deblur and examined the non-positive filtered results. We found that the intra-sample distances based on any of the three metrics tested (weighted/unweighted UniFrac and Bray-Curtis dissimilarity) between any of the pipelines did not vary (Supp Fig 7).

\section{Alpha-diversity metrics vary between denoising pipelines}

We next investigated how the number of ASVs/OTUs called in a real dataset differed between processing pipelines. In the soil dataset, DADA2 called 16609 ASVs, UNOISE3 called 11613 ASVs, Deblur called 8270 ASVs and open-reference OTU clustering called 21297 OTUs (Fig 4A). Across all datasets, we found that DADA2 called the most ASVs among the denoising pipelines and that open-reference OTU clustering called the most ASVs/OTUs overall (Fig 4A, 4C, 4E). On average, DADA2 called 727 more ASVs than Deblur and 532 more ASVs than UNOISE3 while open-reference OTU clustering called 3135 more OTUs/ASVs than DADA2. In all datasets, Deblur called the least amount of ASVs/OTUs. Looking at the number of OTUs called per sample, we found that DADA2 did not correlate well with UNOISE3 or openreference OTU clustering and in the soil dataset the correlation between DADA2 and UNOISE3 was not found to be significant $(p=0.054)(\mathbf{F i g} \mathbf{4 B}, \mathbf{4 D}, \mathbf{4 F})$. This is a concerning result as it indicated the possibility of different biological results based on the pipeline that was chosen to process the data.

One interesting result was that, despite DADA2 calling the most total ASVs among the denoising pipelines, it called the least amount per sample (Supp Fig 8-10) and, among denoising 
413 pipelines, UNOISE3 tended to call the most (Supp Fig 8-10). However, open-reference OTU

414 clustering called the most OTUs/ASVs overall per sample among all approaches that were tested

415 with the exception of the soil dataset.

416

417 Computational requirements are vastly different across denoising pipelines

418 Knowing that all three of these pipelines resulted in similar relative abundance profiles on mock

419 communities and small intra-sample distances on real $16 \mathrm{~S}$ communities, we next investigated

420 how the run time and memory usage differed between the denoising approaches. We found that

421 UNOISE3 (4.6 minutes) was 1273 times faster than DADA2 (5834 minutes) and 15 times faster

422 than Deblur (69.3 minutes) at a total read count of 1,926,000 reads evenly distributed across 103

423 samples (Fig 5A). Run times for all pipelines increased as the number of reads per sample

424 increased. Deblur used a static amount of memory $(611 \mathrm{Mb})$ as reads per sample increased,

425 whereas in general the other two pipelines increased in memory usage as the number of reads per

426 sample increased, with the exception of DADA2 run at 1,926,000 reads (Fig 5B). Deblur used

427 the smallest amount of memory at the maximum read count of $1,926,000$ reads. We found that

428 DADA2 had the highest amount of memory usage (4071 Mb at 1,287,000 reads) among the three

429 pipelines. Interestingly, this usage was more than the amount used at the maximum read count

$430(3600 \mathrm{Mb})$. In addition, none of the runs exceeded the $4 \mathrm{~Gb}$ memory cap on the 32-bit free

431 academic version of USEARCH10.

432

433 Discussion

434 Despite the differences between the underlying algorithms, all four approaches were 435 comparable based on weighted UniFrac and Bray-Curtis distances. However, the tools varied 436 greatly when looking at unweighted or raw number of ASVs/OTUs found. It should be noted that all 
437 denoising pipelines were run using their recommended settings, limiting our comparison to only looking 438 at the default settings for each pipeline. Adjusting of parameters within each sequence analysis pipeline 439 would allow users to tailor towards more sensitivity and specificity as desired. Furthermore, each package 440 uses individual chimera filtering methods which may affect the overall accuracy of the entire pipeline.

441 During mock community data processing, no denoising approach consistently called

442 more ASVs than another, however open-reference OTU clustering found more OTUs than any of 443 the three denoising approaches before abundance filtering (Fig 1). In many cases, the number of

444 OTUs found was vastly greater than the number of expected sequences, which is consistent with

445 previous literature reporting that OTU clustering tends to exaggerate the number of unique 446 organisms found within a sample (Edgar, 2017). No pipeline was able to call all expected 447 sequences for any of the mock communities at 100\% identity (Fig 1), which indicates that some 448 may not have been present or that preparation steps show large bias towards specific 16S rRNA 449 gene variants within an organism. Each sequence processing pipeline was able to detect every 450 organism in the HMP community (note $S$. aureus and $S$. epidermidis are collapsed together as 451 they have the same sequenced region) and the Zymomock community. However, one odd result 452 was that observed abundances within the HMP community were comparable to the expected 453 abundances within the HMP community, but not the Zymomock community (Fig 2A,2D). This again most likely indicates bias during library preparation but could also be due to similar bias among pipelines. In the Extreme dataset, all denoising pipelines missed P. buccalis, $C$. methylpentusum and P. sp._D13 (Fig 2B,SSupp Table 4). All three of these organisms had very

457 low expected abundances (less than $0.00427 \%$ ) which may explain why they were difficult to 458 detect (Supp Table 4). We do not believe it was an issue with sequence depth as the single 459 sample in the mock community was sequenced at a depth of 11.6 million reads and open460 reference OTU clustering only missed one organism (C. methylpentusum). Deblur and UNOISE3 
461 both failed to detect 9 of the 27 expected taxa in the Extreme dataset at 97\% identity, which were

462 all detected by DADA2 and open-reference OTU clustering. Again, these nine organisms were at

463 very low abundances (less than 0.05\%) (Supp Table 4). As mentioned above, open-reference

464 OTU clustering did find 2 more expected taxa than DADA2, however, this came at the cost of

465 significantly increased numbers of non-reference sequences being detected. Differences in

466 detection between DADA2 and the other two denoising pipelines suggests that it is better at

467 detecting organisms that are very rare without the cost of finding significantly more non-

468 reference hits that plagues the open-reference OTU clustering pipeline. Whether finding rare

469 organisms is truly advantageous is debatable, as many of these low-abundance organisms would

470 be removed by typical filtering cut-offs and/or contribute little to weighted beta-diversity metrics

471 such as UniFrac or Bray-Curtis dissimilarity.

472 In all mock datasets, a large number of Unmatched OTUs were found by open-reference

473 OTU clustering and, in some of the datasets, different denoising pipelines also found a relatively

474 large amount of Unmatched ASVs (Fig 1). This could have been due to errors in sequencing or

475 mutations during PCR amplification of the 16S rRNA gene as most sequences did show over

$47690 \%$ identity to the expected sequences, indicating that they originated from a $16 \mathrm{~S}$ rRNA gene

477 (Supp Fig 1-3). It is also possible that many of these sequences are contaminants from the

478 environment, while others may have been from chimeras that were not detected by each

479 pipelines' individual chimera-checking algorithms. We also found a high proportion of matches

480 to the reference database but not the expected sequences. There are several reasons why this

481 could have occurred. Some of these sequences may be contaminants introduced during mock

482 community preparation or sequencing as they match with $100 \%$ identity to a sequence within the

483 Greengenes database indicating that they are a real 16S rRNA gene sequence. However, due to 
484 different pipelines identifying different numbers of these unexpected sequences, it could also 485 suggest that some of these contaminants are likely either chimeric sequences that can be found 486 within the Greengenes database, or sequencing errors/mutations during PCR amplification that 487 could not be corrected for by the respective pipelines. Unsurprisingly, the highest sequence depth 488 mock community (Extreme) had the most Unmatched OTUs (Fig 1), most likely due to the 489 increased absolute abundance of sequencing errors and the increased likelihood of finding minor 490 contaminants. Furthermore, DADA2 found a significantly greater amount of sequences under or 491 at $75 \%$ sequence identity, when compared to the other denoising pipelines, highlighting the 492 trade-off that it makes to find lower-abundance ASVs (Supp Fig 2). In all cases, the open-reference OTU clustering had greatly increased numbers of OTUs 494 found when compared to ASVs found by denoising pipelines (Fig 1). To try and address this 495 issue, we applied an abundance filter of $0.1 \%$ minimum abundance (except for the Extreme 496 community $0.0004 \%$ minimum abundance) to all four different pipelines over all the datasets 497 (Supp Fig 4). The largest effect that this filter had was the reduction of the number of OTUs 498 called by the open-reference OTU clustering. These significant changes in the number of OTUs 499 by open-reference OTU clustering highlight the importance of removing low-abundance OTUs 500 during analysis and demonstrate the stability of denoising approaches after abundance filtering. 501 The filter cutoff did have an impact on the number of Unmatched ASVs called by DADA2 in the 502 HMP mock community (Supp Fig 4A) and the Unmatched ASVs called by Deblur in the 503 Zymomock community (Supp Fig 4E), but had little effect on the number of ASVs called by 504 UNOISE3 on these communities, indicating UNOISE3's tendency to call ASVs of higher 505 abundance. No differences were seen in the Extreme community on ASV output by denoising 506 approaches, most likely due the relatively low cutoff of $0.0004 \%$. Overall, results on the mock 
507 communities showed that Deblur tended to call the least amount of ASVs/OTUs among all

508 pipelines and open-reference OTU clustering called the most.

509 The relative abundances determined for each study on the mock communities were

510 similar to each other irrespective of which pipeline processed the data (Fig 2). This finding

511 suggests that biological conclusions based on microbial relative abundance data should be

512 unaffected by the choice of denoising pipeline. One trend that was noticed in the relative

513 abundance data was that UNOISE3 tended to call higher abundances of non-reference

514 ASVs/OTUs in each mock community except for the Extreme community where open-reference

515 OTU clustering found the highest abundance of non-reference hits. Interestingly, the lowest

516 identity match for any of these ASVs called by UNOISE3 in both the Zymomock and HMP

517 mock communities was still found to be above 90\% identity to the expected sequences (Supp

518 Fig 1,3) and was classified as Gammaproteobacteria by the RDP classifier using a 70\%

519 confidence threshold, suggesting it is a 16S rRNA sequence that may have been introduced by

520 contamination, sequencing bleed-through or acquired an error early on in PCR amplification.

521

The relative abundances determined within the Zymomock and fungal communities were

522 highly similar between pipelines, but markedly differed from the expected result. This finding

523 suggests that either the expected abundances of sequences from these communities may be

524 incorrect or all four pipelines are similarly biased. This non-agreement could also be due to steps

525 during the sequencing processes such as PCR amplification, which may be causing primer bias

526 (Aird et al., 2011) or the inclusion of contaminant organisms. In the case of the fungal

527 community, it is possible that none of these approaches work well with ITS1 data which are

528 more variable than $16 \mathrm{~S}$ data. Additional fungal mock communities should be analyzed in the

529 future to better explore this issue. 
531 communities can be useful, however, they tend to lack the diversity that is found in many real

532 533 534

sample datasets. To address this issue, we compared resulting microbial compositions from each pipeline across three real datasets (exercise, human-associated, and soil). Weighted UniFrac, unweighted UniFrac and Bray-Curtis dissimilarity distances between the same biological samples for each approach were examined. In both cases the weighted UniFrac and Bray-Curtis intra-sample distances between all pipelines for all three datasets were small (less than a median of 0.21) (Fig 3, Supp Figure 5). This complemented our previous results, showing that each pipeline had comparable microbial compositions for the mock communities. Furthermore, plotting the samples on a PCoA or NMDS resulted in the same biological samples from each pipeline grouping together (Fig 3, Supp Figure 5). This indicated that a similar plot would be observed whether the researcher was using the Deblur, UNOISE3, DADA2 or open-reference OTU clustering. Interestingly, Deblur did not agree with the DADA2 or UNOISE3 as much as they agreed with each other on multiple occasions, based on Bray Curtis dissimilarity at the genus level (Supp Fig 5A,5B). This result is interesting, as one of the main differences between Deblur and the other two denoising pipelines is its positive filtering feature, and so we expected this feature to be driving this difference. However, when we compared the other three approaches to Deblur and Deblur without positive filtering, we found no difference (Sup Fig 7). Due to the similar weighted UniFrac results (Fig 3) between the denoising pipelines, we believe that this difference is most likely due to highly similar sequences being classified into slightly different genera.

Comparing the three denoising pipelines to the open-reference OTU picking pipeline, we found that Deblur agreed the least with the OTU pipeline of all the denoising pipelines (Supp 
553 Fig 5). In most cases, OTUs had similar intra-sample distances between pipelines in both

554 weighted UniFrac and Bray Curtis dissimilarity as denoising pipelines had amongst each other.

555 The relatively small differences seen in the abundance-based metrics indicates that choice of

556 pipeline would have minimal impact when looking at weighted beta-diversity metrics. Although,

557 due to denoising pipelines capability of single nucleotide resolution, they may provide more

558 strain information than OTU clustering at $97 \%$ would.

559 Unweighted UniFrac beta-diversity metrics were highly variable between pipelines

560 indicating different bias between pipelines when determining low abundance sequence (Supp

561 Fig6A,6B,6C). When pipelines were plotted together on a PCoA plot we found that the samples

562 separated by approach, rather than sample, indicating that interpretation from unweighted

563 UniFrac data would most likely be impacted based on the pipeline chosen to process any set of

564 given data. We wish to highlight, however, that recent evidence has emerged that the unweighted

565 version of UniFrac analysis can give misleading results (Wong et al. 2016), therefore these

566 patterns should be interpreted with caution.

567

To follow up on the vastly different unweighted UniFrac profiles of samples, we looked

568 at the alpha-diversity between the same samples run by each pipeline and the total number of

569 ASVs/OTUs called by each pipeline in the real datasets. We found that DADA2 called the most

570 ASVs among all denoising approaches, but overall open-reference OTU clustering found the

571 most (Fig 4). This was not surprising, as it agreed with the analysis of the mock communities.

572 Interestingly, despite DADA2 finding the most ASVs overall for all three denoising pipelines, it

573 found the least amount of ASVs per sample (Supp Fig 8-10). We suspect this is due to

574 DADA2's ability to create pooled error profiles and then pick ASVs sample by sample. Overall,

575 open-reference OTU clustering found more OTUs per sample than ASVs found by any denoising 
576 pipelines. This indicated that the number of different organisms found within a sample is directly

577 impacted by the choice of processing pipeline, emphasizing the difficulty in determining the true

578 number of different organisms a sample contains. It should be noted that all of the denoising

579 pipelines provide parameters that could be altered to increase or decrease sensitivity in

580 identifying rare/spurious ASVs depending on a user's targeted application. One alarming result

581 we found was the poor correlation between the number of observed OTUs/ASVs found by

582 DADA2 and both open-reference OTU clustering and UNOISE3 (Fig 4). This is concerning as

583 major differences in biological signal would be seen depending on the approach that was chosen

584 to process the data (i.e. a sample could have relatively low numbers of ASVs based on DADA2

585 analysis, but have relatively high numbers of ASVs/OTUs based on UNOISE3 or an OTU

586 analysis). This issue highlights that the approaches are all very good at identifying highly

587 abundant sequences but vary when identifying low-abundance sequences which will impact

588 metrics that do not take into account the abundance of OTUs/ASVs.

589 A major difference between the three denoising pipelines was their computational run

590 time. UNOISE3 was magnitudes faster than both DADA2 and Deblur. This is most likely due to

591 both the programming language that UNOISE3 is implemented in $(\mathrm{C}++)$, as well as its simple

592 one-pass denoising pipeline. DADA2 was the slowest pipeline and, although computation time

593 could be inconvenient for those with limited computational power, it did not reach times that

594 were impractical even when running almost 2 million total reads. Memory usage for each

595 program also did not reach impractical amounts when running close to 2 million reads, with

596 DADA2 using a maximum amount of $1024 \mathrm{Mb}$ of memory which is a reasonable amount for

597 modern computers. Memory usage by UNOISE3 did not come close to reaching the $4 \mathrm{~Gb}$

598 memory cap on the 32-bit version, even after running 103 samples at a total read depth of 2 
599 million reads. This suggests that for most moderately sized $16 \mathrm{~S}$ datasets the 32 bit version of 600 UNOISE3 should be sufficient.

601

\section{Conclusion}

603

In conclusion, all four pipelines are comparable when looking at weighted results that are

604 based on the relative abundances of ASVs/OTUs while using default settings. However, the 605 approaches do vary when looking at the number of ASVs/OTUs found and unweighted metrics such as unweighted UniFrac, while using the default settings for each denoising pipeline. The

607

608

609

610

611

612

613

614 615

616

617

618

619

620

621

number of ASVs called did not differ between denoising pipelines in a consistent way across mock communities, suggesting that determining species richness within low-diverse samples could be problematic. However, we did find that open-reference OTU clustering consistently called more OTUs than ASV-calling pipelines. Analysis of the real datasets showed that DADA2 consistently called more ASVs than the other two denoising pipelines and that, again, openreference OTU clustering called the most overall. More importantly, in the soil dataset and in the Extreme dataset DADA2 and the open-reference OTU clustering pipeline were capable of finding more low-abundance organisms, but DADA2 could do this without the cost of significantly increasing the number of non-reference hits. The most alarming result was the poor correlation in the number of ASVs/OTUs per sample found between DADA2 and UNOISE3 or open-reference OTU clustering. From this, we believe that choice of approach will have large impacts on determining the alpha-diversity between different samples. Looking at computational run time, we found that DADA2 was by far the slowest denoising pipeline, whereas UNOISE3 was the fastest, processing datasets more than 1200 times faster than DADA2. In the end, the choice of approach did not play a large role in weighted analyses based on microbial abundances, 
622 but did have implications on unweighted results and alpha-diversity metrics. We believe this is

623 promising, as it indicates that no matter the choice of approach, a similar weighted biological

624 signal will be seen. On the other hand, extreme caution is required when looking at unweighted

625 results and alpha-diversity metrics between different pipelines.

626

627 Acknowledgements

628

629 We would like to thank members of the Langille lab for providing feedback and suggestions for 630 additional analysis during lab meetings. We would especially like to thank Karl Leuschen for 631 providing previous data on a comparison between QIIME OTU clustering and DADA2 632 denoising.

633

634

635

636 References

637

638 Aird, D., Ross, M. G., Chen, W.-S., Danielsson, M., Fennell, T., Russ, C., Jaffe, D. B., 639 Nusbaum, C., \& Gnirke, A. (2011). Analyzing and minimizing PCR amplification bias in 640 Illumina sequencing libraries. Genome Biology, 12(2), R18. https://doi.org/10.1186/gb$641 \quad 2011-12-2-r 18$

642 Allali, I., Arnold, J. W., Roach, J., Cadenas, M. B., Butz, N., Hassan, H. M., Koci, M., Ballou, 643 A., Mendoza, M., Ali, R., \& Azcarate-Peril, M. A. (2017). A comparison of sequencing 
644 platforms and bioinformatics pipelines for compositional analysis of the gut microbiome.

645 BMC Microbiology, 17(1), 194. https://doi.org/10.1186/s12866-017-1101-8

646 Altschul, S. F., Gish, W., Miller, W., Myers, E. W., \& Lipman, D. J. (1990). Basic local

647 alignment search tool. Journal of Molecular Biology, 215(3), 403-410.

$648 \quad$ https://doi.org/10.1016/S0022-2836(05)80360-2

649 Amir, A., McDonald, D., Navas-Molina, J. A., Kopylova, E., Morton, J. T., Zech Xu, Z.,

650 Kightley, E. P., Thompson, L. R., Hyde, E. R., Gonzalez, A., \& Knight, R. (2017). Deblur

651 Rapidly Resolves Single-Nucleotide Community Sequence Patterns. mSystems, 2(2).

652 https://doi.org/10.1128/mSystems.00191-16

653 Bokulich, N. A., Rideout, J. R., Mercurio, W. G., Shiffer, A., Wolfe, B., Maurice, C. F., Dutton,

654 R. J., Turnbaugh, P. J., Knight, R., \& Caporaso, J. G. (2016). mockrobiota: A Public

655 Resource for Microbiome Bioinformatics Benchmarking. mSystems, 1(5).

656 https://doi.org/10.1128/mSystems.00062-16

657 Callahan, B. J., McMurdie, P. J., \& Holmes, S. P. (2017). Exact sequence variants should replace 658 operational taxonomic units in marker-gene data analysis. The ISME Journal, 11(12), 2639-

659 2643. https://doi.org/10.1038/ismej.2017.119

660 Callahan, B. J., McMurdie, P. J., Rosen, M. J., Han, A. W., Johnson, A. J. A., \& Holmes, S. P. 661 (2016). DADA2: High resolution sample inference from Illumina amplicon data. Nature $662 \quad$ Methods, 13(7), 581-583. https://doi.org/10.1038/nmeth.3869

663 Caporaso, J. G., Bittinger, K., Bushman, F. D., DeSantis, T. Z., Andersen, G. L., \& Knight, R. 664 (2010a). PyNAST: A flexible tool for aligning sequences to a template alignment.

665 Bioinformatics, 26(2), 266-267. https://doi.org/10.1093/bioinformatics/btp636 
666 Caporaso, J. G., Kuczynski, J., Stombaugh, J., Bittinger, K., Bushman, F. D., Costello, E. K., 667 Fierer, N., Peña, A. G., Goodrich, J. K., Gordon, J. I., Huttley, G. A., Kelley, S. T., Knights, 668 D., Koenig, J. E., Ley, R. E., Lozupone, C. A., McDonald, D., Muegge, B. D., Pirrung, M., 669 Reeder, J., Sevinsky, J. R., Turnbaugh, P. J., Walters, W. A., Widmann, J., Yatsunenko, T., 670 Zaneveld, J., \& Knight, R. (2010b). QIIME allows analysis of high-throughput community 671 sequencing data. Nature Methods, 7, 335. http://dx.doi.org/10.1038/nmeth.f.303

672 Cole, J. R., Wang, Q., Fish, J. A., Chai, B., McGarrell, D. M., Sun, Y., Brown, C. T., Porras673 Alfaro, A., Kuske, C. R., \& Tiedje, J. M. (2014). Ribosomal Database Project: Data and 674 tools for high throughput rRNA analysis. Nucleic Acids Research, 42(Database issue), 675 D633-D642. https://doi.org/10.1093/nar/gkt1244

676 Comeau, A. M., Douglas, G. M., \& Langille, M. G. I. (2017). Microbiome Helper: A Custom 677 and Streamlined Workflow for Microbiome Research. mSystems, 2(1).

678 https://doi.org/10.1128/mSystems.00127-16

679 680 681 682 683 684 685 686 687
DeSantis, T. Z., Hugenholtz, P., Larsen, N., Rojas, M., Brodie, E. L., Keller, K., Huber, T., Dalevi, D., Hu, P., \& Andersen, G. L. (2006). Greengenes, a chimera-checked 16S rRNA gene database and workbench compatible with ARB. Applied and Environmental Microbiology, 72(7), 5069-5072. https://doi.org/10.1128/AEM.03006-05

Douglas, G. M., Hansen, R., Jones, C. M. A., Dunn, K. A., Comeau, A. M., Bielawski, J. P., Tayler, R., El-Omar, E. M., Russell, R. K., Hold, G. L., Langille, M. G. I., \& Van Limbergen, J. (2018). Multi-omics differentially classify disease state and treatment outcome in pediatric Crohn's disease. Microbiome, 6(1), 13. https://doi.org/10.1186/s40168-018-0398-3 
688 Edgar, R. C. (2010). Search and clustering orders of magnitude faster than BLAST.

689 Bioinformatics, 26(19), 2460-2461. http://dx.doi.org/10.1093/bioinformatics/btq461

690 Edgar, R. C. (2016). UNOISE2: Improved error-correction for Illumina 16S and ITS amplicon 691 sequencing. bioRxiv. http://biorxiv.org/content/early/2016/10/15/081257.abstract

692 Edgar, R. C. (2017). Accuracy of microbial community diversity estimated by closed- and open693 reference OTUs. PeerJ, 5, e3889. https://doi.org/10.7717/peerj.3889

694 Edgar, R. C., Haas, B. J., Clemente, J. C., Quince, C., \& Knight, R. (2011). UCHIME improves 695 sensitivity and speed of chimera detection. Bioinformatics, 27(16), 2194-2200.

696 https://doi.org/10.1093/bioinformatics/btr381

697 Fierer, N., \& Jackson, R. B. (2006). The diversity and biogeography of soil bacterial 698 communities. Proceedings of the National Academy of Sciences of the United States of

699 America, 103(3), 626-631. https://doi.org/10.1073/pnas.0507535103

700

701

702

703

704

705

706

707

708

709

Illumina. (n.d.). Effects of Index Misassignment on Multiplexing and Downstream Analysis. https://www.illumina.com/content/dam/illuminamarketing/documents/products/whitepapers /index-hopping-white-paper-770-2017-004.pdf

Kõljalg, U., Nilsson, R. H., Abarenkov, K., Tedersoo, L., Taylor, A. F. S., Bahram, M., Bates, S. T., Bruns, T. D., Bengtsson-Palme, J., Callaghan, T. M., Douglas, B., Drenkhan, T., Eberhardt, U., Dueñas, M., Grebenc, T., Griffith, G. W., Hartmann, M., Kirk, P. M., Kohout, P., Larsson, E., Lindahl, B. D., Lücking, R., Martín, M. P., Matheny, P. B., Nguyen, N. H., Niskanen, T., Oja, J., Peay, K. G., Peintner, U., Peterson, M., Põldmaa, K., Saag, L., Saar, I., Schüßler, A., Scott, J. A., Senés, C., Smith, M. E., Suija, A., Taylor, D. L., Telleria, M. T., Weiss, M., \& Larsson, K.-H. (2013). Towards a unified paradigm for 
710

711

712 Lamoureux, E. V, Grandy, S. A., \& Langille, M. G. I. (2017). Moderate Exercise Has Limited

713 but Distinguishable Effects on the Mouse Microbiome. mSystems, 2(4), e00006-17.

714 https://doi.org/10.1128/mSystems.00006-17

715 Martin, M. (2011). Cutadapt removes adapter sequences from high-throughput sequencing reads.

716 EMBnet.journal; Vol 17, No 1: Next Generation Sequencing Data Analysis.

717 http://dx.doi.org/10.14806/ej.17.1.200

718 MIRARAB, S., NGUYEN, N., \& WARNOW, T. (2011). SEPP: SATé-Enabled phylogenetic

719 placement. In Biocomputing 2012 (pp. 247-258). WORLD SCIENTIFIC.

720

721

722

723

724

725

726

727 Pruesse, E., Quast, C., Knittel, K., Fuchs, B. M., Ludwig, W., Peplies, J., \& Glöckner, F. O.

728

729

730

731 https://doi.org/doi:10.1142/9789814366496_0024

Oksanen, J., Blanchet, F. G., Friendly, M., Kindt, R., Legendre, P., McGlinn, D., ... Wagner, H. (2018). vegan: Community ecology package. Retrieved from https://cran.rproject.org/package $=$ vegan

Plummer, E., \& Twin, J. (2015). A Comparison of Three Bioinformatics Pipelines for the Analysis of Preterm Gut Microbiota using 16S rRNA Gene Sequencing Data. Journal of Proteomics \& Bioinformatics, 8(12). https://doi.org/doi: 10.4172/jpb.1000381 (2007). SILVA: A comprehensive online resource for quality checked and aligned ribosomal RNA sequence data compatible with ARB. Nucleic Acids Research, 35(21), 7188-7196. https://doi.org/10.1093/nar/gkm864

R Development Core Team. (2008). R: A Language and Environment for Statistical Computing. 
Vienna, Austria. http://www.r-project.org

733

734

735

736

737

738

739

740

741

742

743

744

745

746

747

748

749

750

751

752

753

754

\section{9}

Rognes, T., Flouri, T., Nichols, B., Quince, C., \& Mahé, F. (2016). VSEARCH: A versatile open source tool for metagenomics. PeerJ, 4, e2584. https://doi.org/10.7717/peerj.2584

Tange, O. (2011). GNU Parallel: the command-line power tool. The USENIX Magazine, 36(1), 42-47. https://doi.org/10.5281/zenodo.16303

Wickham, H. (2009). ggplot2: Elegant Graphics for Data Analysis. Springer-Verlag New York. http://ggplot2.org

Wong, R.G., Wu, J.R., \& Gloor, G.B. (2016). Expanding the UniFrac Toolbox. PLoS ONE, 11, e0161196.

Yurgel, S. N., Douglas, G. M., Comeau, E. M., Mammoliti, M., Dusault, A., Percival, D., \& Langille, M. G. I. (2017). Variation in Bacterial and Eukaryotic Communities Associated with Natural and Managed Wild Blueberry Habitats. Phytobiomes, 1, 102-113. https://doi.org/10.1094/PBIOMES-03-17-0012-R

Zhang, J., Kobert, K., Flouri, T., \& Stamatakis, A. (2014). PEAR: A fast and accurate Illumina Paired-End reAd mergeR. Bioinformatics, 30(5), 614-620.

https://doi.org/10.1093/bioinformatics/btt593

(1)


Table $\mathbf{1}$ (on next page)

Qualitative Comparison of DADA2, Deblur, and UNOISE3 
1 Table 1:

2 Qualitative Comparison of DADA2, Deblur, and UNOISE3

3

4

\begin{tabular}{|c|c|c|c|c|c|c|c|}
\hline Pipeline & $\begin{array}{c}\text { Implemented } \\
\text { In }\end{array}$ & $\begin{array}{c}\text { Open } \\
\text { Source }\end{array}$ & $\begin{array}{c}{ }^{*} \text { Pooled } \\
\text { Sampling }\end{array}$ & $\begin{array}{c}{ }^{* *} \text { Positive } \\
\text { Filtering }\end{array}$ & $\begin{array}{c}\text { Version } \\
\text { Tested }\end{array}$ & $\begin{array}{c}\text { GUI via } \\
\text { QIIME2 }\end{array}$ & $\begin{array}{c}\text { Publication } \\
\text { Date }\end{array}$ \\
\hline DADA2 & R & Yes & Yes & No & 1.6 & Yes & April 13 2016 \\
\hline Deblur & Python & Yes & No & Yes & 1.0 .2 & Yes & $\begin{array}{c}\text { March 7, } \\
2017\end{array}$ \\
\hline UNOISE3 & C++ & No & Yes & No & 3 & No & Oct 15, 2016 \\
\hline
\end{tabular}

5

$6 *$ When all sequences from all samples are denoised at the same time (in contrast to running each 7 sample separately).

8 ** Compares resulting ASVs to a database (Greengenes for Deblur) and discards reads if they do 9 not match a certain identity threshold (88\% for Deblur). 


\section{Figure 1 (on next page)}

Total number of ASVs/OTUs identified by each sequence processing method for four different mock communities.

Amplicon sequence variants/Operational taxonomic units (ASVs/OTUs) were compared to a database of full-length amplicon sequences for just the microbes supposedly in the community ("Expected") and against the full SILVA or ITS databases ("Database") using BLASTN at $97 \%$ and $100 \%$ identity cut-offs. "Unmatched" sequences did not match an expected sequence or the SILVA/TSS databases at $97 \%$ identity or greater. Dotted lines indicate the total number of ASVs/OTUs expected, accounting for $16 \mathrm{~S}$ copy variation within genomes. Note that the $y$-axis for open-reference OTU clustering is different than the $y$-axis on the denoising methods. A) Human Microbiome Project equal abundance mock community; B) Extreme dataset; C) Fungal ITS1 mock community; D) Zymomock community. 


\section{Figure 2 (on next page)}

Relative abundances of taxa generated by each sequence processing method for four different mock communities.

All ASVs/OTUs that matched with expected sequences at $97 \%$ or greater identity were assigned taxonomy using a BLASTN search against the expected sequences provided for the Extreme, Human Microbiome Project, and Zymomock mock communities. All ASVs/OTUs that matched an expected species with $97 \%$ or greater identity to the UNITE database were classified as expected sequences in the fungal mock community. Non-reference refers to the abundance of ASVs/OTUs that did not match expected sequences with $97 \%$ or greater identity. A) Human Microbiome Project equal abundance mock community; B) Extreme dataset - it is important to note that some organisms are not displayed in this figure due to their very low abundances; C) Fungal ITS1 mock community; D) Zymomock community. 


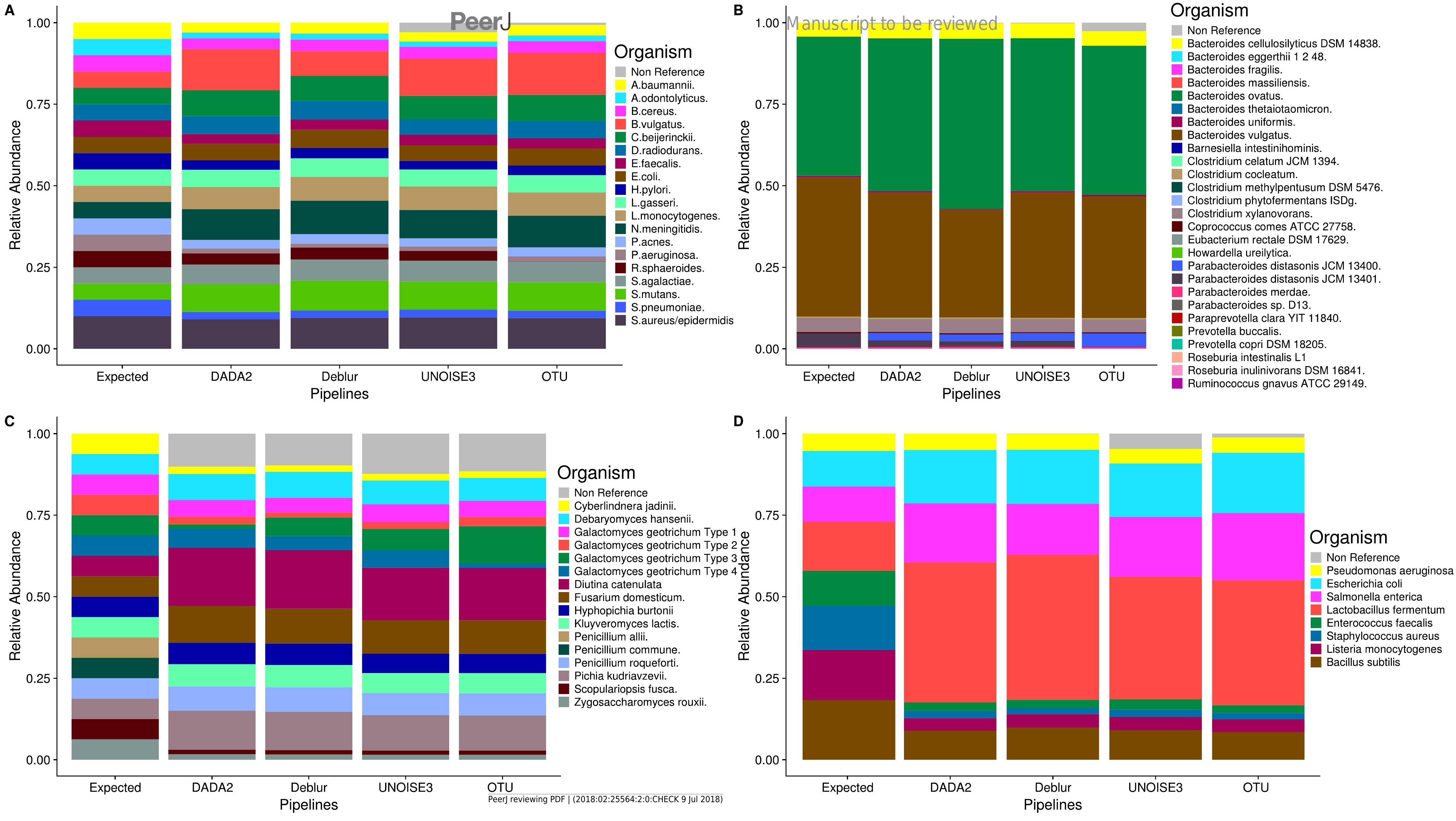




\section{Figure 3 (on next page)}

Weighted UniFrac intra-sample distances between sequence processing methods based on three real datasets.

A-C) The weighted UniFrac distances between the same biological samples based on ASVs/OTUs outputted by each of the different sequence processing methods on the soil, human associated and Exercise datasets, respectively. D-F) Principal coordinates analysis of the weighted UniFrac distances of all the samples in the soil, human associated, and Exercise datasets, respectively. The four different sample profiles generated for each biological sample are colour-coded and are joined by an interconnecting line. 


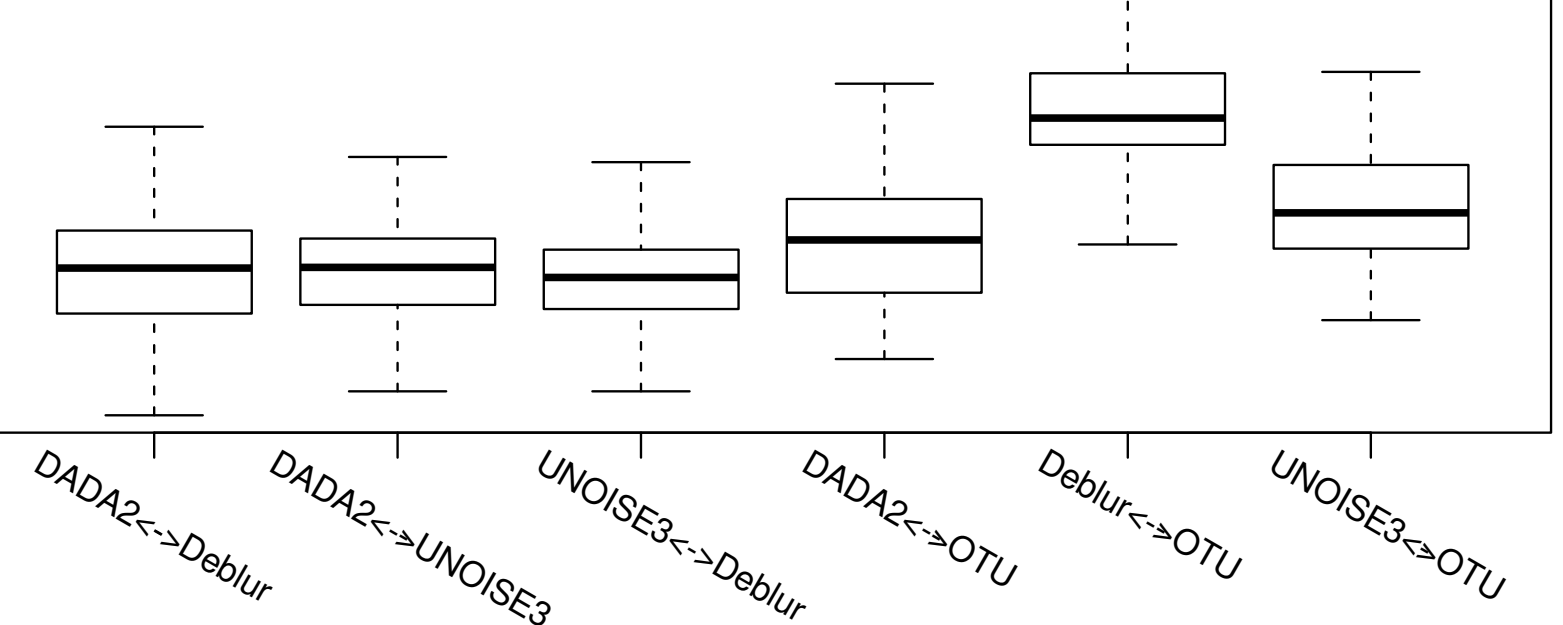

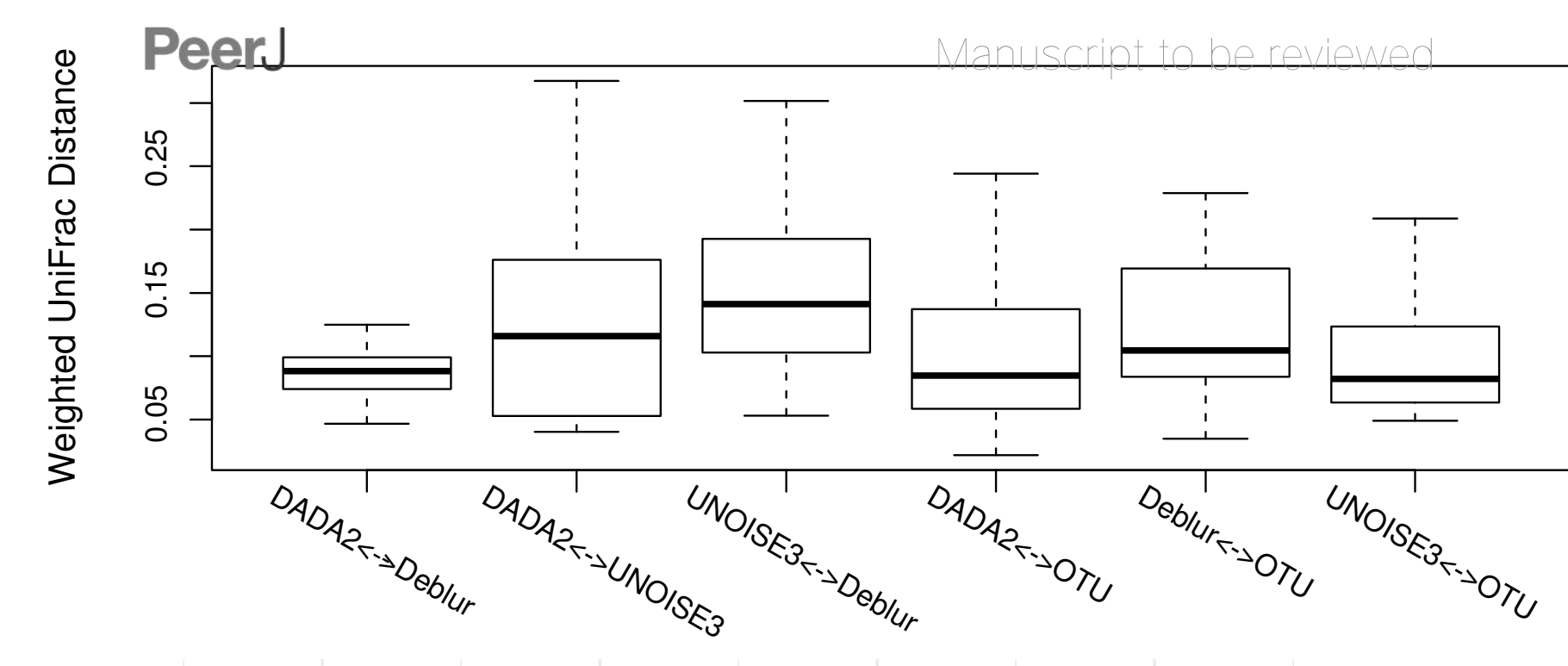

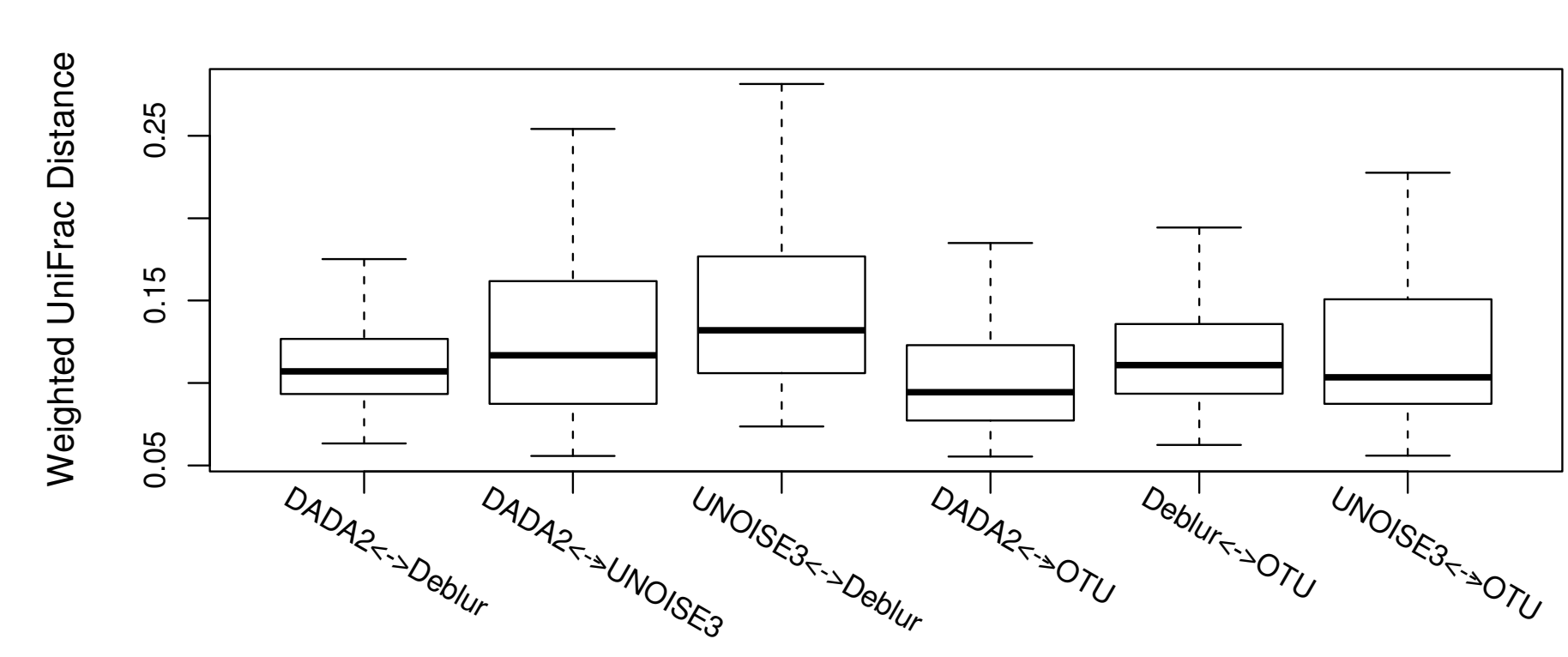
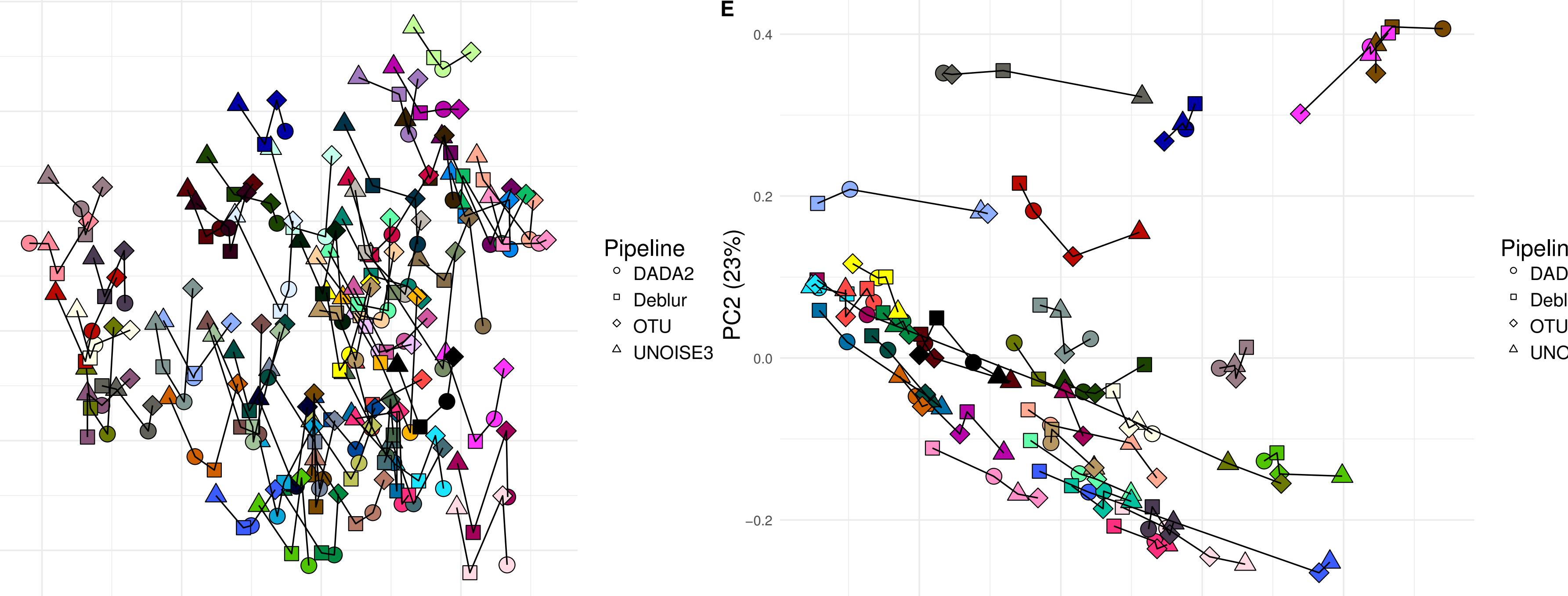

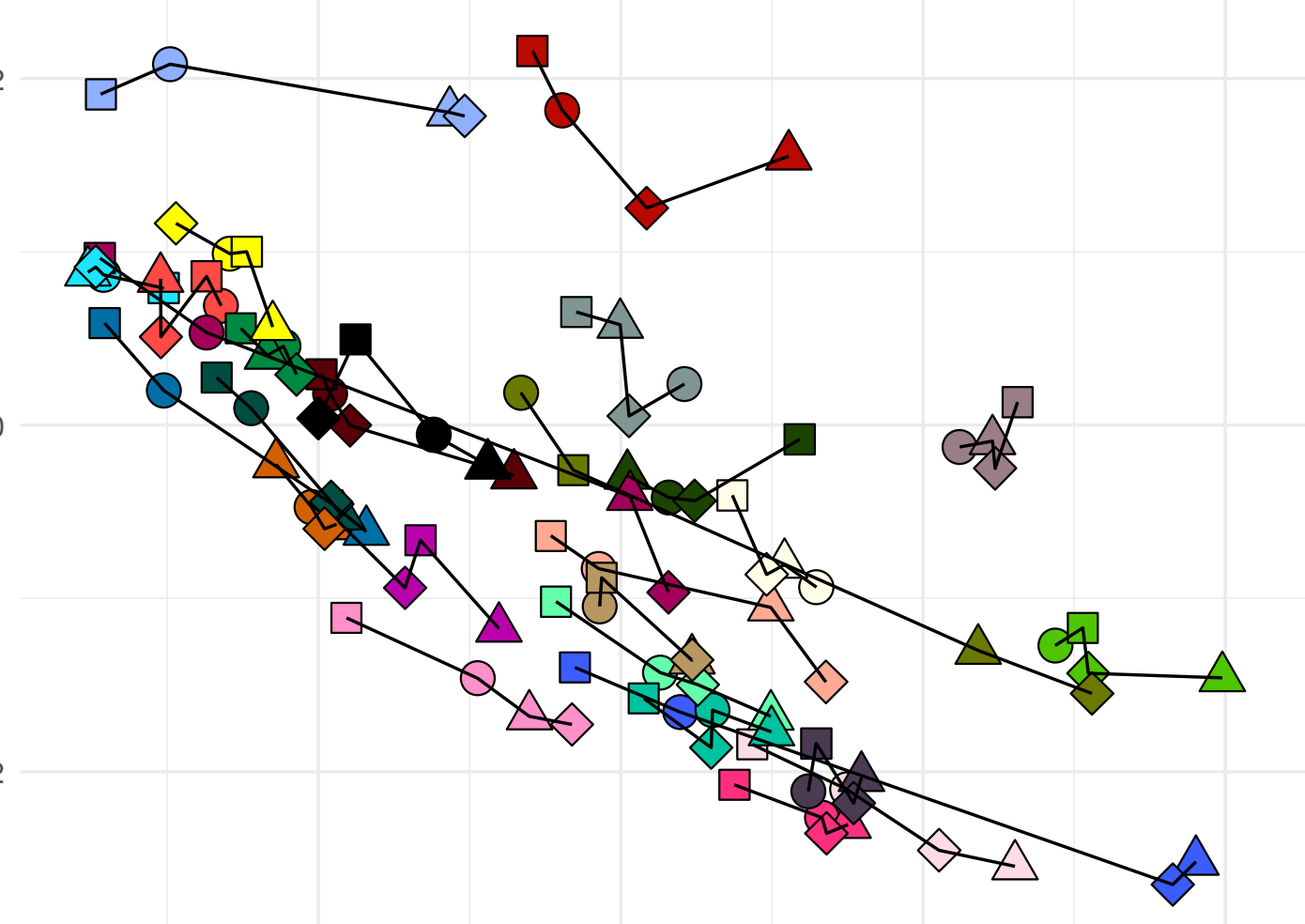

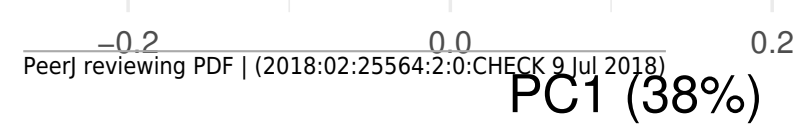

Pipeline
o DADA2 \begin{tabular}{l}
- DADA2 \\
- Deblur \\
- OTU \\
\hline
\end{tabular}

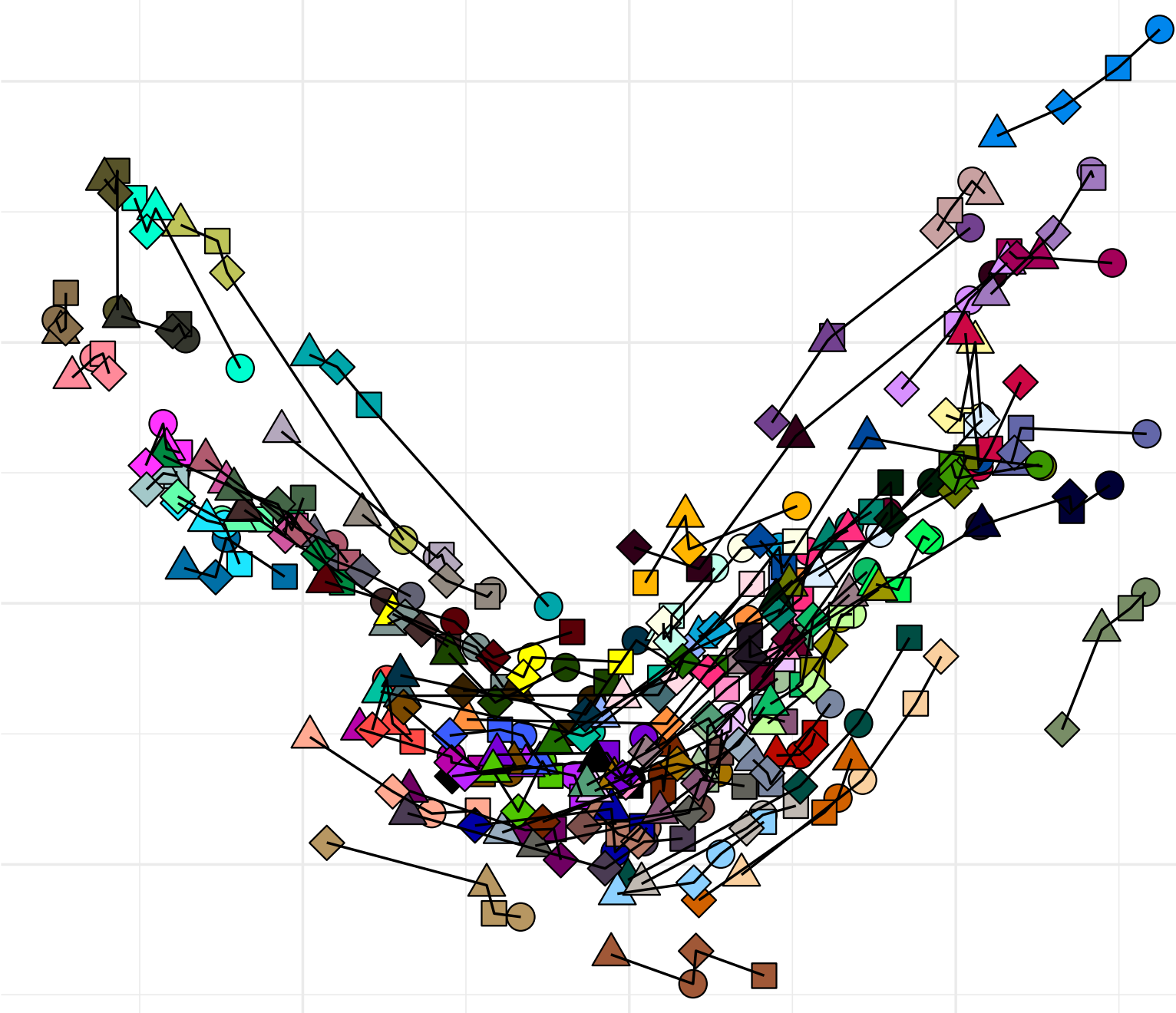


Figure 4 (on next page)

Total number of ASVs/OTUs called by each processing method and the per-sample observed ASV/OTUs correlation between each sequence processing method.

$A, C, E)$ The total numbers of ASVs/OTUs determined by each method on the soil, Exercise, and human associated datasets, respectively. B,D,F) Heatmaps of the Spearman correlations between the numbers of observed ASVs/OTUs per sample between different sequence processing methods. Significant $p$-values $(p<0.05)$ are indicated by *. 
Figure 5 (on next page)

Run time and memory usage of each denoising pipeline on a dataset of varying size

The time in seconds A) and memory in megabytes B) to run varying amounts of reads through the three different denoising methods. Note time is on a $\log _{10}$ scale. 
